\title{
Adjuvant goserelin and ovarian preservation in chemotherapy treated patients with early breast cancer: results from a randomized trial
}

\author{
A. Sverrisdottir $\cdot$ M. Nystedt $\cdot$ H. Johansson $\cdot$ \\ T. Fornander
}

Received: 27 October 2008/ Accepted: 6 January 2009/Published online: 20 January 2009

(C) Springer Science+Business Media, LLC. 2009

\begin{abstract}
The purpose of this randomized study was to examine if goserelin concomitant to CMF-chemotherapy as adjuvant treatment for premenopausal breast cancer, protects the ovaries from premature failure. A total of 285 premenopausal breast cancer patients, in a randomized adjuvant trial (Zoladex in premenopausal patients (ZIPP)), were assigned to a study on ovarian function. Node positive patients were assigned to CMF-(cyclophosphamide, methotrexate and 5-fluorouracil) chemotherapy in addition to endocrine therapy. All patients were randomly assigned to receive 2 years of goserelin, goserelin plus tamoxifen, tamoxifen alone or no endocrine treatment. We studied, if menses were affected in the treatment groups, up to 36 months after randomization. One year after completed CMF- and endocrine therapy, $36 \%$ of the women in the goserelin group reported menses, compared to $7 \%$ in the goserelin plus tamoxifen group, $13 \%$ in the tamoxifen group and $10 \%$ of the controls. Among women treated with goserelin, there was a statistically significant increase in the proportion of menstruating women, 1 year after completed treatment compared to at 24 months of treatment $(P=0.006)$, in contrast to all other treatment groups, who were unchanged or more often amenorrheic. In our study, there is some evidence of protective effect of goserelin on ovarian function in CMF treated women. This effect was not observed in the combined tamoxifen and goserelin treatment.
\end{abstract}

A. Sverrisdottir - M. Nystedt $\cdot$ H. Johansson - T. Fornander Departments of Oncology, Karolinska Institute and University Hospital, Stockholm, Sweden

A. Sverrisdottir $(\bowtie)$

Landspitali University Hospital, Reykjavik, Iceland

e-mail: asgerds@landspitali.is
Keywords Breast cancer - Amenorrhea - Goserelin · Tamoxifen · Chemotherapy

\section{Introduction}

Premature ovarian failure (POF) and infertility is a frequent long-term result of cytotoxic chemotherapy, in young women with breast cancer [1-3]. The risk of ovarian failure is highly correlated to the woman's age, type of drug, dosage and duration $[1,4,5]$. In most studies, the majority of women over 40 years of age become amenorrheic after chemotherapy and the chances of restoring their ovarian function are meagre [6].

Approximately $25 \%$ of the women with breast cancer are premenopausal at the time of diagnosis [7]. As the incidence of breast cancer is rising, the number of young women diagnosed with the disease is increasing [8]. Lately, it has become more common for women to delay family building and childbirth, and it is not unusual for women over the age of 40 to consider pregnancy. To avoid signs and symptoms of early menopause is an even more frequently reported request of this group of patients. Pregnancies after breast cancer do not adversely affect the prognosis $[9,10]$ and should in general not be discouraged. Young age at diagnosis appears to be an independent risk factor for recurrence or metastatic disease [11] and young patients' tumours tend to be more aggressive. Therefore, adjuvant cytotoxic chemotherapy is often recommended for this group of patients. Earlier studies have shown higher rates of amenorrhea from CMF compared to anthracycline-based therapy, but the additional effect of anthracyclines or taxanes in comparable duration, dose-dense or dose-intense schemes is not clear [12-15]. 
Treatment induced ovarian ablation, whether by endocrine- or chemotherapy, radiation or surgery, results in increased disease free survival and overall survival in premenopausal endocrine responsive breast cancer [16]. However, it is not clear whether permanent ovarian failure is superior to temporary amenorrhea. There have also been concerns that combined chemo-endocrine treatment might be inferior to sequential treatment [17]. The additional effect of ovarian ablative treatment after chemotherapy has as well been debated and is currently under investigation in several international trials such as the Suppression of Ovarian Function trial (SOFT). So far, recent results from a meta-analysis of randomized luteinizing hormone release hormone agonist (LHRH-a) trials show that combined therapy is not inferior and there is an additive effect from LHRH-a after chemotherapy, at least for women younger than 40 years [18].

Besides infertility, POF has multiple physiologic consequences affecting many organs and increases the risk of other long-term side effects, such as osteoporosis, vasomotor symptoms, cardiovascular disease and sexual dysfunction [2, 3, 12,19-21]. As treatment results improve and more women have excellent prognosis after adjuvant chemotherapy, the demands for information on adverse effects increases and greater effort is needed in attempt to assure physical and psychological well being after treatment. Treatment induced infertility among women desiring children, can account for great psychological stress and should be prevented if possible [22]. Although "prophylactic" fertility treatment by cryopreservation of fertilized ova or ovarian tissue can be offered to some of these patients, such approach is not feasible for all, due to factors such as cancer treatment delay and cost. These approaches are still very experimental and do not restore hormone production for a longer duration. Ovarian protection by endocrine manoeuvring could therefore render an important therapeutic option in prevention of POF, in young women with malignant disease, where adjuvant cytotoxic chemotherapy is recommended.

In the literature, there are some reports on the protective effects from an LHRH-a use concomitant to chemotherapy [3, 23-26]. The mechanism behind such a protection is unknown, but may be explained by LHRH-a acting by suppression of the pituitary-gonadal axis, resulting in an inactive or resting state of the ovaries which might preserve primordial follicles from permanent cytotoxic damage. However, there is to our knowledge, no data from randomized studies of ovarian effects of LHRH-a in addition to chemotherapy and tamoxifen in breast cancer patients. The present study was designed as a sub-protocol to a randomized adjuvant trial (Zoladex In Premenopausal Patients (ZIPP)), to examine the effects of the LHRH-a goserelin on menstrual status, when used concomitant to CMF-chemotherapy, with or without tamoxifen.

\section{Patients and methods}

The ZIPP study was a collaboration of four randomized multi-center trials (Stockholm Breast Cancer Study Group, Sweden, SE Sweden Breast Cancer Group, Cancer Research UK and UCL Cancer Trials centre, UK and Gruppo Interdisciplinare Valutazione Interventi in Oncologia, Italy) and has been described elsewhere [27]. In the Stockholm centers, node positive women were allocated to adjuvant CMF chemotherapy (six cycles of cyclophsophamide $600 \mathrm{mg} / \mathrm{m}^{2}$, methotrexate $40 \mathrm{mg} / \mathrm{m}^{2}$ and 5-fluorouracil $600 \mathrm{mg} / \mathrm{m}^{2}$ intravenously days 1 and 8 , every 28 days), in addition to endocrine therapy. Patients operated on with breast conserving surgery received postoperative radiotherapy $50 \mathrm{~Gy}$ through 5 weeks, to the breast. Patients with four or more positive lymph nodes received additionally loco regional radiotherapy including the chest wall, axillary- and supraclavivular lymph nodes 46 Gy through 4.5 weeks. The dose of tamoxifen was $40 \mathrm{mg}$ /day orally and goserelin $3.6 \mathrm{mg}$ subcutaneously every 28 days. For goserelin, every injection was documented in the patient records. The treatment duration for both tamoxifen and goserelin was 2 years and was given irrespective of hormone receptor status, according to the inconclusive data at the time of study. Endocrine treatment was given concomitant to chemotherapy. The randomization was stratified in three groups: node negative patients receiving no chemotherapy, patients with 1-3 positive lymph nodes receiving chemotherapy, and patients with four or more positive lymph nodes receiving both chemotherapy and loco-regional radiotherapy (Fig. 1). Randomization was carried out by telephone to a central office where the patient identifiers were recorded before the allocated treatment was revealed to the responsible physician. Treatment allocation was based on balanced lists using the permuted block technique.

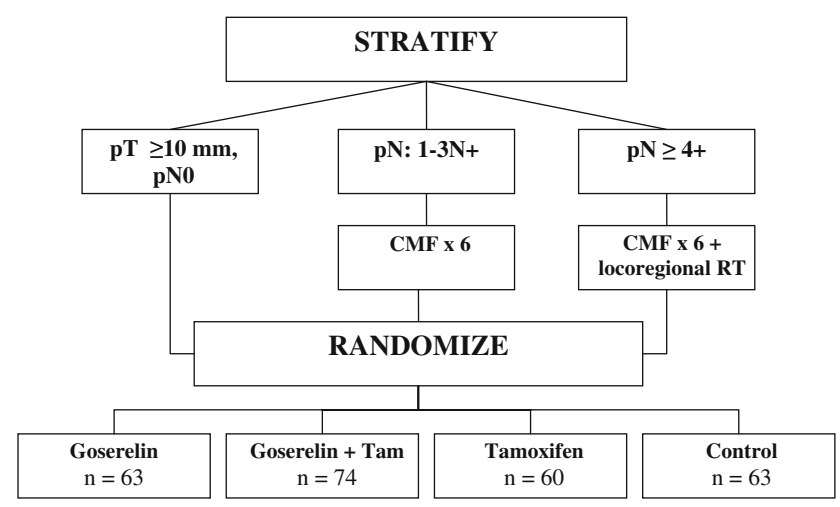

Fig. 1 Study design. All patients with breast conserving surgery were assigned radiotherapy (RT) to the breast, 50 Gy's in 5 weeks. Abbreviations: pT: pathological tumour size in millimetres, $\mathrm{pN}$ : pathological nodal status, CMF: cyclophosphamide, methotrexate and 5-fluorouracil. Tam: tamoxifen 
Within the ZIPP-trial, the Stockholm Breast Cancer Study Group initiated sub-studies of treatment related side effects, such as effects on ovarian function, bone mineral density, return to work and quality of life. Between October 1990 and June 1994, 285 out of 408 (70\%) eligible patients in the randomized trial were recruited to the study of ovarian function. Patients were allocated to the study before randomization to the treatment arms. The inclusion criteria were invasive breast cancer $\geq 10 \mathrm{~mm}$, premenopausal menstrual status (defined as last menstruation $<6$ months earlier), primary surgery consisting of a mastectomy or a sector resection plus axillary node dissection and no clinical evidence of distant metastases. The exclusion criteria were inoperable breast cancer, prior radiotherapy or neo-adjuvant chemotherapy and prior or concurrent endocrine therapy. Self-reporting questionnaires were obtained from patients at scheduled visits at the outpatient clinic at baseline (before randomization), 3 months, 12, 24, 30 and 36 months after randomization. The study was designed and conducted according to WHO's Good Clinical Practice guidelines and was approved by the Karolinska Institute Regional Ethics Committee.

\section{Instrument}

The questions regarding menses were a part of the questionnaire designed for the ZIPP sub-study of side effects as has been reported [20]. The women reported, either if menses had ceased, had not ceased but become scanty or not ceased, since prior survey and visit to the outpatient clinic. The first survey was made at the time of randomization and the time-period since prior survey, varied from 3 months up to 9 months.

\section{Statistical methods}

All analyses were performed according to the intention-totreat principle. In the analyses for each time point, menses self reported as regular or irregular were assessed as having menstruations. Patients reporting absence of menses were assessed as amenorrheic. Patients with recurrent disease were excluded from the analysis. The analyses are presented as results at baseline, 3 months, 12, 24, 30 and 36 months. Fisher's exact test was used to assess differences between the treatment groups, as shown in "two by two tables".

\section{Results}

Baseline characteristics were well balanced as listed in Table 1. Recruitment of patients and distribution to different treatment arms is presented in Fig. 2.
A total of 285 (70\%) out of 408 eligible patients were recruited for the study of ovarian function. The 123 patients not recruited, was due to patient's preference. Additionally twenty-five patients were excluded because of recurrent disease. The remaining 260 patients were included in the analyses. Self-reporting questionnaires were obtained from patients before randomization to endocrine treatment up to 36 months after randomization. Information on menstrual status was missing from four patients at baseline, twelve patients at 3 months and additionally 39 patients up to 36 months.

Tolerability study of this cohort has shown that treatment with goserelin and tamoxifen resulted in reversible menopausal symptoms whereas CMF treated experienced symptoms throughout the study period as has been reported [20].

\section{Amenorrhea}

\section{CMF chemotherapy treated}

At baseline, 6\% (7/119) women reported absence of menses, which was in accordance to inclusion criteria, as premenopausal status was defined at last menses within less than 6 months. At 3 months, 50\% (13/26) of the women in the control group reported amenorrhea. For women receiving tamoxifen (T), the figure was 73\% (19/26). Women treated with goserelin (G) or goserelin plus tamoxifen (GT), reported amenorrhea in 93-94\% (26/28 and $34 / 36$ respectively) of cases. At 24 months, i.e. after 2 years of endocrine treatment, $85 \%(17 / 20)$ of the controls, 95\% (19/20) of the T group, 97\% (29/30) of the GT and $92 \%(22 / 24)$ of the G group were amenorrheic. At this time point, there was no statistically significant difference in amenorrhea for goserelin treated compared to all other treatment groups $(P=1.00)$ (Table 2). Six months after cessation of endocrine treatment, the proportion of amenorrheic women continued to increase to $94 \%(16 / 17)$ for the controls, was $87 \%$ (20/23) for the T group, unchanged $92 \%(24 / 26)$ for the GT group, but only $67 \%$ (14/21) of the women treated with goserelin reported absence of menses at this time point.

At 36 months, i.e. 1 year after stopped endocrine treatment, the proportion of amenorrheic women in the control group was $90 \%$ (18/20), 87\% (20/23) for the T group, $93 \%$ (27/29) for the GT group but had decreased to 64\% (14/22) for the $\mathrm{G}$ group. The proportion of menstruating women in the $\mathrm{G}$ group increased statistically significant, compared to all other groups where menses were unchanged or decreasing between 24 and 36 months $(P=0.006)$ (Table 2 and Fig. 3). 
Table 1 Patient characteristics

\begin{tabular}{|c|c|c|c|c|}
\hline Endocrine treatment & $\begin{array}{l}\text { Goserelin } \\
n=63\end{array}$ & $\begin{array}{l}\text { Goserelin + Tam } \\
n=74\end{array}$ & $\begin{array}{l}\text { Tamoxifen } \\
n=60\end{array}$ & $\begin{array}{l}\text { Control } \\
n=63\end{array}$ \\
\hline CMF treated $(\%)$ & $29(46)$ & $37(50)$ & $29(48)$ & $28(44)$ \\
\hline Age $<45$ years & $10(34)$ & $12(32)$ & $10(34)$ & $11(39)$ \\
\hline Age $\geq 45$ years & $19(66)$ & $25(68)$ & $19(66)$ & $17(61)$ \\
\hline \multicolumn{5}{|l|}{ Age in years } \\
\hline Median (range) all & $45(29-54)$ & $45(29-54)$ & $45(29-53)$ & $46(36-55)$ \\
\hline CMF & $45(36-51)$ & $46(35-54)$ & $45(29-53)$ & $45(29-53)$ \\
\hline Not CMF & $46(36-55)$ & $45(29-52)$ & $45(33-52)$ & $45(32-54)$ \\
\hline \multicolumn{5}{|l|}{ Tumour size } \\
\hline$<20 \mathrm{~mm}$ & 29 & 37 & 30 & 29 \\
\hline$>20 \mathrm{~mm}$ & 34 & 35 & 30 & 34 \\
\hline Unknown & 0 & 2 & 0 & 0 \\
\hline \multicolumn{5}{|l|}{ Nodal status } \\
\hline Positive & 29 & 37 & 28 & 29 \\
\hline Negative & 34 & 37 & 32 & 34 \\
\hline \multicolumn{5}{|l|}{ ER status } \\
\hline Positive & 45 & 45 & 45 & 46 \\
\hline Negative & 12 & 21 & 9 & 11 \\
\hline Unknown & 6 & 8 & 6 & 6 \\
\hline \multicolumn{5}{|l|}{ PgR status } \\
\hline Positive & 41 & 49 & 41 & 40 \\
\hline Negative & 16 & 17 & 13 & 15 \\
\hline Unknown & 6 & 8 & 6 & 8 \\
\hline \multicolumn{5}{|l|}{ Surgery } \\
\hline Partial mastectomy & 42 & 43 & 33 & 35 \\
\hline Mastectomy & 21 & 31 & 27 & 28 \\
\hline
\end{tabular}

\section{Not CMF treated}

All randomized patients reported having menses at baseline.

At 3 months, 9\% (3/34) of the controls, 21\% (6/29) of the T group, $86 \%(31 / 36)$ of the GT group and 97\% (32/33) of the $\mathrm{G}$ group were amenorrheic. At 2 years after randomization, $13 \%$ (4/31) of the controls, $21 \%(5 / 24)$ of the T-group, $69 \%(22 / 32)$ of GT-group and $82 \%$ (23/28) of the G-group reported amenorrhea. Six months after completed endocrine treatment, $17 \%(5 / 29)$ of the controls, $12 \%$ $(3 / 24)$ of the T group, $28 \%(8 / 29)$ of the GT group and $37 \%$ $(10 / 27)$ of the $G$ group were amenorrheic. At 36 months, i.e. 1 year after completed endocrine treatment, $20 \%$ (6/30 and 5/25 respectively) of the controls and $\mathrm{T}$ group, $32 \%(10 / 31)$ of the GT group and $41 \%(12 / 29)$ of the $G$ group were amenorrheic. At 36 months, i.e. 1 year after stopped endocrine therapy, there was no statistical significant difference in menstrual status of women treated with goserelin compared to all other treatment groups $(P=$ 0.15) (Fig. 3).

\section{Discussion}

This was a randomized adjuvant study of LHRH-a effects on ovarian function in breast cancer patients treated with chemotherapy with or without concomitant tamoxifen. Our study shows that amenorrhea is significantly less frequent one year after completed treatment among patients treated with goserelin as the only additive endocrine therapy during chemotherapy. This suggests that goserelin has a role in protecting ovarian function in cytotoxic milieu as has been reported in several non-randomized studies [24-26, 28-33]. The size of this effect is nevertheless moderate and may be due to several effect modifiers in our study. In the present study, the age is rather high for a premenopausal population, with a mean of 45 years. This is an age where natural menopause approaches and age above 40 years is undisputedly a strong risk factor for permanent chemotherapy induced amenorrhea [34].

The timing of goserelin treatment is another factor, which possibly may not be optimal concerning a potential effect on preservation of ovarian function. Theoretically, 


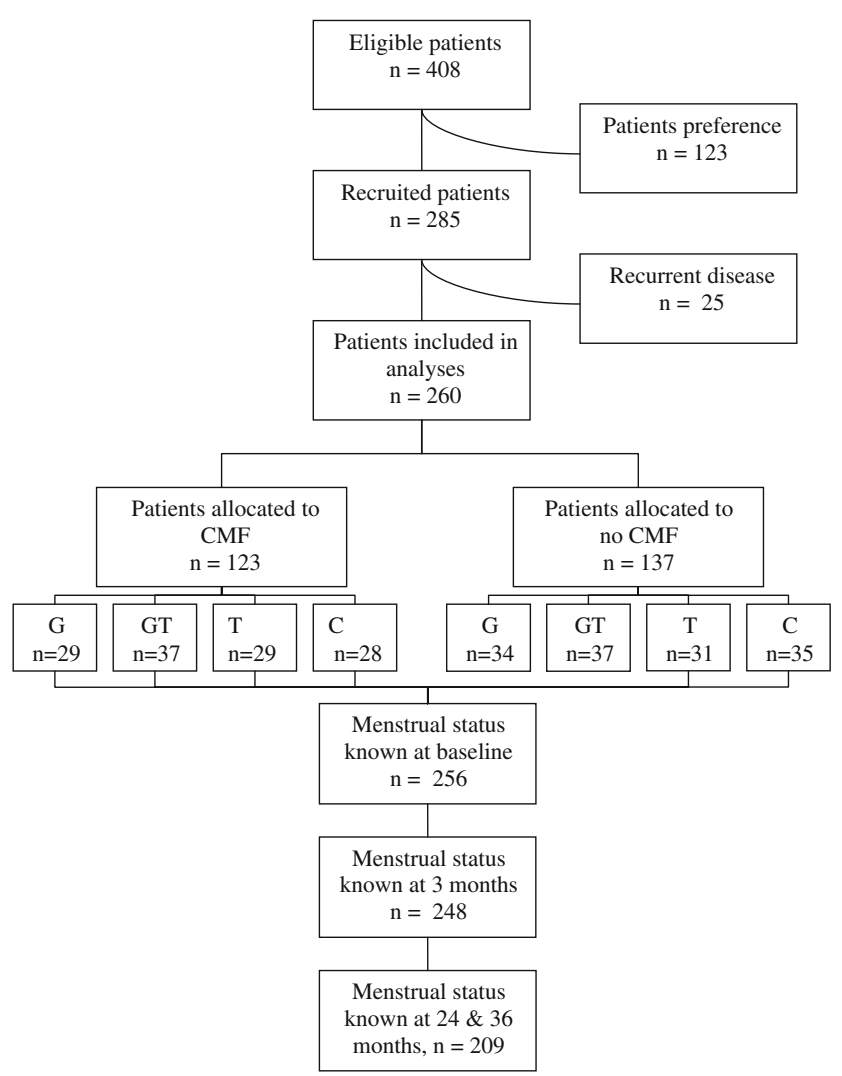

Fig. 2 Patients recruitment and compliance to study protocol. Abbreviations: CMF: cyclophosphamide, methotrexate, fluorouracil, G: goserelin, GT: goserelin plus tamoxifen, T: tamoxifen, C: control

LHRH-a treatment should preferably start at least 2 weeks before initiation of chemotherapy as an approach to optimise ovarian suppression in advance of cytotoxic chemotherapy effects. In our study, however, goserelin injections were started simultaneously as the first CMF course, which may be too late for achieving full effect.
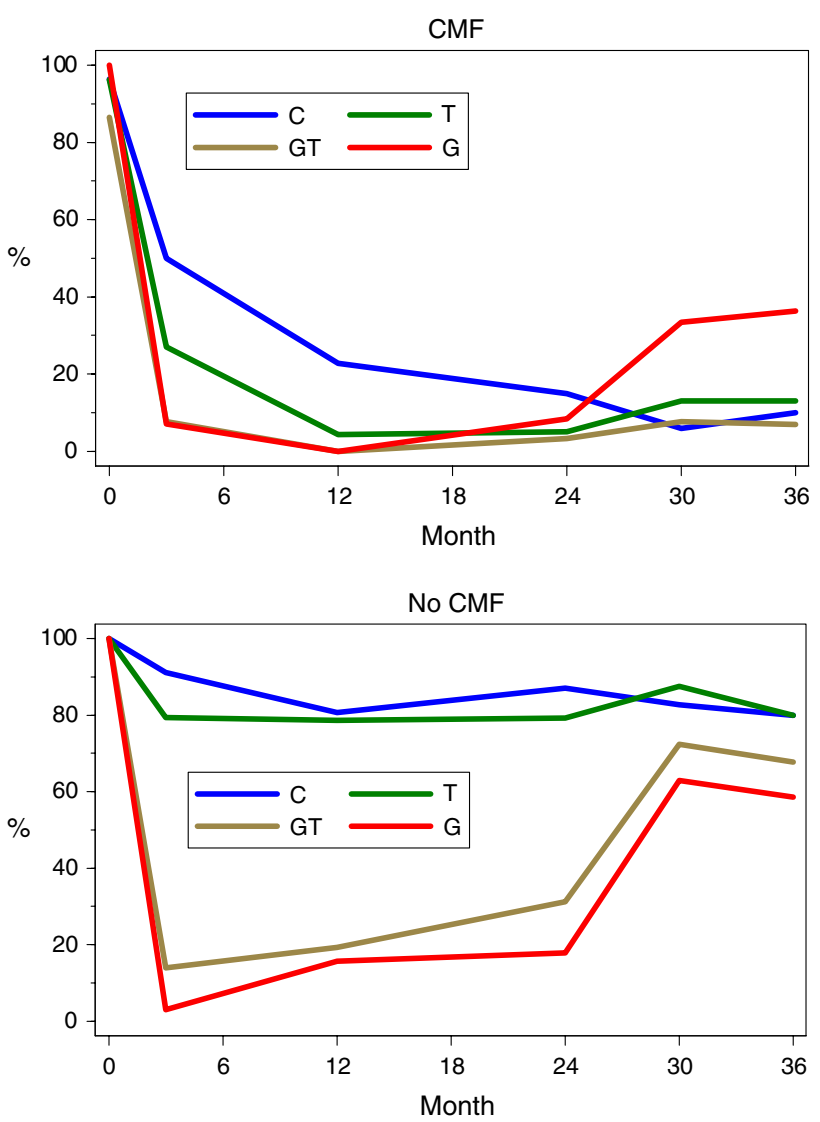

Fig. 3 Ovarian function according to treatment. Percentage of women menstruating at different time points. C: control, T: tamoxifen, GT: goserelin plus tamoxifen, G: goserelin

When tamoxifen was added to goserelin in CMF treated women, there was no statistical significant change in the proportion of menstruating women, 1 year after stopped treatment, in contrast to the CMF treated women receiving goserelin alone. The mechanism behind this is unclear but

Table 2 Menstrual status in CMF-chemotherapy treated women

\begin{tabular}{|c|c|c|c|c|c|c|c|c|}
\hline \multirow[t]{2}{*}{ Menses } & \multicolumn{2}{|l|}{$\mathrm{G}$ versus $\mathrm{C}$} & \multicolumn{2}{|l|}{$\mathrm{G}$ versus $\mathrm{T}$} & \multicolumn{2}{|c|}{ G versus TG } & \multicolumn{2}{|c|}{$\mathrm{G}$ versus all other } \\
\hline & G & $\mathrm{C}$ & $\mathrm{G}$ & $\mathrm{T}$ & $\mathrm{G}$ & TG & G & $\mathrm{C}, \mathrm{T}$ or $\mathrm{TG}$ \\
\hline \multicolumn{9}{|c|}{ After 2 years of endocrine treatment } \\
\hline Yes & $2(8 \%)$ & $3(15 \%)$ & $2(8 \%)$ & $1(5)$ & $2(8 \%)$ & $1(3 \%)$ & $2(8 \%)$ & $5(7 \%)$ \\
\hline No & $22(92 \%)$ & $17(85 \%)$ & $22(92 \%)$ & $19(95 \%)$ & $22(92 \%)$ & $29(97 \%)$ & $22(92 \%)$ & $65(93 \%)$ \\
\hline Total & 24 & 20 & 24 & 20 & 24 & 30 & 24 & 70 \\
\hline Fishers exact & $P=0.65$ & & $P=1.00$ & & $P=0.58$ & & $P=1.00$ & \\
\hline \multicolumn{9}{|c|}{ One year after stopped endocrine treatment } \\
\hline Yes & $8(36 \%)$ & $2(10 \%)$ & $8(36 \%)$ & $3(13 \%)$ & $8(36 \%)$ & $2(7 \%)$ & $8(36 \%)$ & $7(10 \%)$ \\
\hline No & $14(64 \%)$ & $18(90 \%)$ & $14(64 \%)$ & $20(87 \%)$ & $14(64 \%)$ & $27(93 \%)$ & $14(64 \%)$ & $65(90 \%)$ \\
\hline Total & 22 & 20 & 22 & 23 & 22 & 29 & 22 & 72 \\
\hline Fishers exact & $P=0.071$ & & $P=0.091$ & & $P=0.013$ & & $P=0.006$ & \\
\hline
\end{tabular}

$G$ goserelin, $T$ tamoxifen, $C$ control, $G T$ goserelin plus tamoxifen 
can be due to tamoxifen obscuring the goserelin effect possibly by its oestrogen agonistic properties.

The use of menstruations as a sole surrogate for ovarian function might be regarded as a limitation of our study whereas measurements of follicle stimulating hormone (FSH), luteinizing hormone (LH), estradiol and antMüllerian hormone have been reported by Anderson et al., to be a more precise assessment [35]. However, a previous study by Poikonen et al., showed that post-chemotherapy menstrual status is a clinically useful marker of menstrual status and FSH and LH less reliable [36]. The evaluation of serum markers such as FSH and LH after chemotherapy is therefore challenging and furthermore not appropriate during the period of goserelin treatment. Tamoxifen is as well reported to affect the gonadotrophins by decreasing FSH and LH levels [37, 38].

Possible confounders, such as the previous use of contraceptive pills as well as hormone replacement therapy, due to menopausal symptoms, were not examined, but are expected to be evenly distributed between treatment arms in a randomized study like this.

Our results are in accordance with several earlier studies where CMF frequently induces early and irreversible amenorrhea $[1,3,12,39]$. CMF was standard chemotherapy at the time of the study, whereas anthracycline based chemotherapy, with or without taxanes, have recently become more commonly used. The extent of ovarian suppression from these agents is not well defined but seems nevertheless substantial [34]. Current trials investigating goserelin prophylaxis during taxane-based chemotherapy, such as the ZORO trial by the German Breast Group, may clarify this effect. A meta-analysis of randomized adjuvant trials has recently shown no therapeutic benefit with combination endocrine therapy versus tamoxifen or goserelin alone [18]. If these findings will be repeated in an ongoing and extended up-date of the ZIPP-trial, it may turn out that goserelin alone is equally as effective treatment as in combination with tamoxifen or tamoxifen alone except for the possibility of ovarian protection.

In conclusion, chemotherapy, ovarian ablation and tamoxifen improve survival in hormone receptor positive premenopausal breast cancer. The present study shows that the addition of LHRH-a goserelin alone may as well prevent permanent amenorrhea, for some women receiving cytotoxic chemotherapy. Young breast cancer patients should therefore not only receive adequate information on possible long-term risks of chemotherapy therapy but should as well be considered for the option of goserelin treatment when reversible amenorrhea is strongly desired. The results of this study must, however, be interpreted cautiously because of several limitations in design and data collection and need to be confirmed in future randomized trials before any change in clinical routine is justified.
Acknowledgements We thank nursing staff at the Breast cancer units, Department of Oncology, Karolinska Hospital for their excellent work coordinating patients' visits, tests and questionnaires. This study was supported by the Swedish Cancer Society.

\section{References}

1. Bines J, Oleske DM, Cobleigh MA (1996) Ovarian function in premenopausal women treated with adjuvant chemotherapy for breast cancer. J Clin Oncol 14(5):1718-1729

2. Bernhard J et al (2007) Adjuvant chemotherapy followed by goserelin compared with either modality alone: the impact on amenorrhea, hot flashes, and quality of life in premenopausal patients-the International Breast Cancer Study Group Trial VIII. J Clin Oncol 25(3):263-270. doi:10.1200/JCO.2005.04.5393

3. Jonat $\mathrm{W}$ et al (2002) Goserelin versus cyclophosphamide, methotrexate, and fluorouracil as adjuvant therapy in premenopausal patients with node-positive breast cancer: the Zoladex Early Breast Cancer Research Association Study. J Clin Oncol 20(24):4628-4635

4. Goodwin PJ et al (1999) Risk of menopause during the first year after breast cancer diagnosis. J Clin Oncol 17(8):2365-2370

5. Padmanabhan N, Rubens RD, Howell A (1986) Adjuvant chemotherapy in early breast cancer. Lancet 328(8519):1333-1334. doi:10.1016/S0140-6736(86)91459-5

6. Oktay K et al (2003) Fertility preservation in breast cancer patients: IVF and embryo cryopreservation after ovarian stimulation with tamoxifen. Hum Reprod 18(1):90-95. doi:10.1093/ humrep/deg045

7. Jemal A et al (2006) Cancer statistics, 2006. CA Cancer J Clin 56(2):106-130

8. Socialstyrelsen (2005) Cancer incidence in Sweden 2005, Stockholm

9. von Schoultz E et al (1995) Influence of prior and subsequent pregnancy on breast cancer prognosis. J Clin Oncol 13(2): 430-434

10. Ives A et al (2007) Pregnancy after breast cancer: population based study. BMJ 334(7586):194. doi:10.1136/bmj.39035.6671 76.55

11. Goldhirsch A et al (2005) Meeting highlights: international expert consensus on the primary therapy of early breast cancer 2005 . Ann Oncol 16(10):1569-1583. doi:10.1093/annonc/mdi326

12. Shapiro CL, Manola J, Leboff M (2001) Ovarian failure after adjuvant chemotherapy is associated with rapid bone loss in women with early-stage breast cancer. J Clin Oncol 19(14):33063311

13. Fornier MN et al (2005) Incidence of chemotherapy-induced, long-term amenorrhea in patients with breast carcinoma age 40 years and younger after adjuvant anthracycline and taxane. Cancer 104(8):1575-1579. doi:10.1002/cncr.21385

14. Martin $M$ et al (2005) Adjuvant docetaxel for node-positive breast cancer. N Engl J Med 352(22):2302-2313. doi:10.1056/ NEJMoa043681

15. Citron ML et al (2003) Randomized trial of dose-dense versus conventionally scheduled and sequential versus concurrent combination chemotherapy as postoperative adjuvant treatment of node-positive primary breast cancer: first report of Intergroup Trial C9741/Cancer and Leukemia Group B Trial 9741. J Clin Oncol 21(8):1431-1439. doi:10.1200/JCO.2003.09.081

16. Early Breast Cancer Trialists' Collaborative Group (EBCTCG) (2005) Effects of chemotherapy and hormonal therapy for early breast cancer on recurrence and 15-year survival: an overview of the randomised trials. Lancet 365(9472):1687-1717. doi:10.1016/ S0140-6736(05)66544-0 
17. Albain K, Green SJ, Ravdin PM et al (2002) Adjuvant chemohormonal therapy for primary breast cancer should be sequential instead of concurrent: initial results from Intergroup Trial 0100 (SWOG-8814). Proc Am Soc Clin Oncol 21 (abstr 143)

18. LHRH-agonists in Early Breast Cancer Overview Group (2007) Use of luteinising-hormone-releasing hormone agonists as adjuvant treatment in premenopausal patients with hormone-receptorpositive breast cancer: a meta-analysis of individual patient data from randomised adjuvant trials. Lancet 369(9574):1711-1723. doi:10.1016/S0140-6736(07)60778-8

19. Chapman RM, Sutcliffe SB, Malpas JS (1979) Cytotoxic-induced ovarian failure in Hodgkin's disease. II. Effects on sexual function. JAMA 242(17):1882-1884. doi:10.1001/jama.242.17.1882

20. Nystedt $\mathrm{M}$ et al (2003) Side effects of adjuvant endocrine treatment in premenopausal breast cancer patients: a prospective randomized study. J Clin Oncol 21(9):1836-1844. doi:10.1200/ JCO.2003.04.024

21. Sverrisdottir A et al (2004) Bone mineral density among premenopausal women with early breast cancer in a randomized trial of adjuvant endocrine therapy. J Clin Oncol 22(18):3694-3699. doi:10.1200/JCO.2004.08.148

22. Cwikel J, Gidron Y, Sheiner E (2004) Psychological interactions with infertility among women. Eur J Obstet Gynecol Reprod Biol 117(2):126-131. doi:10.1016/j.ejogrb.2004.05.004

23. Chapman RM, Sutcliffe SB, Malpas JS (1979) Cytotoxic-induced ovarian failure in women with Hodgkin's disease. I. Hormone function. JAMA 242(17):1877-1881. doi:10.1001/jama.242.17. 1877

24. Behringer K et al (2005) Secondary amenorrhea after Hodgkin's lymphoma is influenced by age at treatment, stage of disease, chemotherapy regimen, and the use of oral contraceptives during therapy: a report from the German Hodgkin's Lymphoma Study Group. J Clin Oncol 23(30):7555-7564. doi:10.1200/JCO.2005. 08.138

25. Recchia F et al (2002) Goserelin as ovarian protection in the adjuvant treatment of premenopausal breast cancer: a phase II pilot study. Anticancer Drugs 13(4):417-424. doi:10.1097/00001 813-200204000-00011

26. Blumenfeld $\mathrm{Z}$ et al (2002) Fertility after treatment for Hodgkin's disease. Ann Oncol 13(Suppl 1):138-147

27. Baum $M$ et al (2006) Adjuvant goserelin in pre-menopausal patients with early breast cancer: results from the ZIPP study. Eur J Cancer 42(7):895-904. doi:10.1016/j.ejca.2005.12.013

28. Mardesic T et al (2004) Protocol combining GnRH agonists and $\mathrm{GnRH}$ antagonists for rapid suppression and prevention of gonadal damage during cytotoxic therapy. Eur J Gynaecol Oncol 25(1):90-92
29. Urruticoechea A et al (2008) Ovarian protection with goserelin during adjuvant chemotherapy for pre-menopausal women with early breast cancer (EBC). Breast Cancer Res Treat 110(3): 411-416

30. Potolog-Nahari C, Fishman A, Cohen I (2007) Protection of ovarian function and fertility using a combination of gonadotropin-releasing hormone $(\mathrm{GnRH})$ agonist and $\mathrm{GnRH}$ antagonist during cancer treatment in young females. Gynecol Endocrinol 23(5):290-294. doi:10.1080/09513590701327661

31. Franke HR, Smit WM, Vermes I (2005) Gonadal protection by a gonadotropin-releasing hormone agonist depot in young women with Hodgkin's disease undergoing chemotherapy. Gynecol Endocrinol 20(5):274-278. doi:10.1080/09513590400027414

32. Rody A et al (2005) Use of goserelin in the treatment of breast cancer. Expert Rev Anticancer Ther 5(4):591-604. doi:10.1586/ 14737140.5.4.591

33. Pacheco B et al (2001) Use of GnRH analogs for functional protection of the ovary and preservation of fertility during cancer treatment in adolescents: a preliminary report. Gynecol Oncol 81(3):391-397. doi:10.1006/gyno.2001.6181

34. Walshe JM, Denduluri N, Swain SM (2006) Amenorrhea in premenopausal women after adjuvant chemotherapy for breast cancer. J Clin Oncol 24(36):5769-5779. doi:10.1200/JCO.2006. 07.2793

35. Anderson RA et al (2006) The effects of chemotherapy and longterm gonadotrophin suppression on the ovarian reserve in premenopausal women with breast cancer. Hum Reprod 21(10):2583-2592. doi:10.1093/humrep/del201

36. Poikonen $\mathrm{P}$ et al (2000) Prognostic effect of amenorrhoea and elevated serum gonadotropin levels induced by adjuvant chemotherapy in premenopausal node-positive breast cancer patients. Eur J Cancer 36(1):43-48. doi:10.1016/S0959-8049 (99)00225-7

37. Robertson JF et al (1989) Combined endocrine effects of LHRH agonist (Zoladex) and tamoxifen (Nolvadex) therapy in premenopausal women with breast cancer. Br J Surg 76(12):12621265. doi:10.1002/bjs. 1800761213

38. Rossi E et al (2008) Endocrine effects of adjuvant letrozole + triptorelin compared with tamoxifen + triptorelin in premenopausal patients with early breast cancer. J Clin Oncol 26(2):264-270. doi:10.1200/JCO.2007.13.5319

39. Bonadonna $\mathrm{G}$ et al (2005) 30 years' follow up of randomised studies of adjuvant CMF in operable breast cancer: cohort study. BMJ 330(7485):217. doi:10.1136/bmj.38314.622095.8F 